\title{
DEPRESSÃO EM IDOSOS: o papel do profissional farmacêutico
}

DOI: $10.22289 / 2446-922 X . V 4 N 1 A 3$

\author{
Karen Vanessa Ferreira ${ }^{1}$ \\ Nathalya Isabel Melo
}

\section{RESUMO}

A depressão é um dos principais problemas de saúde mental, caracterizada por sintomas como tristeza, desesperança, apatia, indiferença, desinteresse, além de sintomas físicos, como alterações no sono e no apetite. Essa doença pode afetar pessoas de todas as faixas etárias, porém, os idosos requerem uma atenção especial quando comparados às pessoas mais jovens, pelos riscos que os avanços da idade podem trazer. No Brasil, o número de idosos terá um aumento significativo até 2025 , tal fato é preocupante, uma vez que pessoas idosas estão mais suscetíveis a algumas doenças, entre elas, a depressão. A depressão é vista como uma das doenças mais impactantes das últimas décadas, podendo levar ao suicídio. As causas se apresentam de forma variada e podem estar ligadas a fatores biológicos, psicológicos ou sociais. Alguns tipos de doenças, como câncer ou doenças infecciosas, abuso de álcool, perdas de pessoas próximas ou bens materiais, bem como o uso de alguns medicamentos também podem causar sintomas depressivos. $O$ tratamento pode ser feito através de psicoterapia e de medicamentos, os quais passaram por um processo significativo de evolução no decorrer dos anos. Cabe ao farmacêutico, realizar o acompanhamento farmacoterapêutico dos pacientes, garantindo a sua segurança e orientando-os quanto ao uso correto dos medicamentos. No presente trabalho, objetivou-se analisar dados referentes à depressão em pessoas idosas, tendo como foco principal, os tipos de tratamentos disponíveis, assim como os riscos que um antidepressivo pode oferecer à saúde de um idoso. Para o desenvolvimento do estudo, foi feita uma pesquisa bibliográfica qualitativa e descritiva na literatura disponível, acerca dos pontos de maior relevância dentro do tema. A partir das informações obtidas, foi possível concluir que a atenção e capacitação dos profissionais da saúde são imprescindíveis na qualidade do atendimento ao paciente, assim como o cuidado no momento da prescrição do medicamento, já que alguns destes podem causar danos à saúde.

Palavras-chave: Depressão; Idosos; Antidepressivos.

\section{ABSTRACT}

Depression is one of the main mental health problems, characterized by symptoms such as sadness, hopelessness, apathy, indifference, lack of interest, as well as physical symptoms such as changes in sleep and appetite. This disease can affect people of all group age, however, the elderly require special attention when compared to younger people, due to the

\footnotetext{
${ }^{1}$ Endereço eletrônico de contato: karen_vanessa93@hotmail.com

Recebido em 18/10/2017. Aprovado pelo conselho editorial para publicação em 14/11/2017.
}

Rev. Psicol Saúde e Debate. Fev., 2018:4(1):44-60. 
risks that the advancements of age can bring. In Brazil, the number of elderly people will increase significantly by 2025 , a worrying fact, since elderly people are more susceptible to some diseases, among them, depression. Depression is one of the most striking illnesses of the last few decades, which can lead to suicide. The causes are varied and may be linked to biological, psychological or social factors. Some types of ailments such as cancer or infectious diseases, alcohol abuse, loss of dear people or material possessions as well as the use of some medications can also cause depressive symptoms. The treatment can be done through psychotherapy and medication, which have undergone a significant process of evolution over the years. It is up to the pharmacist to perform the pharmacotherapeutic follow-up of the patients, guaranteeing their safety and guiding them as to the correct use of the medicines. In the present study, we aimed to analyze data referring to depression in the elderly, focusing on the types of treatments available, as well as the risks that an antidepressant can offer to the health of an elderly person. For the development of the study, a qualitative and descriptive bibliographical research was done in the available literature about the most relevant points within the theme. From the information obtained, it was possible to conclude that the attention and qualification of health professionals are essential in the quality of patient care, as well as the care at the moment of prescription of the medicine, since some of these can cause health damage.

Keywords: Depression; Elderly; Antidepressant.

\section{INTRODUÇÃO}

A depressão apresenta-se como um distúrbio da área afetiva ou do humor, com impacto funcional em qualquer faixa etária. Na velhice, a depressão engloba os aspectos biológicos (vulnerabilidade na saúde decorrente de doenças crônicas), psicológicos (viuvez, falta de atividades sociais e mudanças de papeis) e sociais (escolaridade, pobreza, solidão e modificações no suporte social). Essa doença psiquiátrica está associada ao maior risco de morbidade e mortalidade, ao aumento na utilização dos serviços de saúde, à negligência no autocuidado, à adesão reduzida aos regimes terapêuticos e a maiores riscos de suicídio (Soares et al. 2013).

As pessoas de idade mais avançada geralmente necessitam de uma atenção especial dos serviços de saúde mental quando comparadas à população de faixas etárias mais jovens, tal fato pode ser explicado pela elevação de riscos que o aumento da idade pode trazer, bem como as repercussões de doenças crônico-degenerativas (Stella, Gobbi, Corazza, \& Costa, 2002).

O tratamento da depressão no idoso, busca amenizar o sofrimento acarretado por essa doença, reduzir o risco de suicídio, melhorar a qualidade de vida do paciente garantindo seu bem-estar geral. Assim como o tratamento de outras doenças neuropsiquiátricas, uma pessoa depressiva requer cuidados especializados. 
Psicoterapia, intervenção psicofarmacológica e eletroconvulsoterapia são estratégias que podem ser utilizadas durante o tratamento (Stella et al., 2002).

Impulsionado pelas informações apresentadas e pelo fato de que o número de pessoas idosas no país tem aumentado significativamente nos últimos anos, e visto ainda que a estimativa é que até 2025, exista no Brasil aproximadamente 32 milhões de idosos, surgiu o objeto de estudo do presente trabalho, com o objetivo de buscar na literatura dados referentes à depressão em idosos, com foco nos tratamentos disponíveis e nos riscos associados ao uso de antidepressivos por idosos (Stella et al., 2002).

O trabalho foi realizado por meio de uma pesquisa bibliográfica de caráter descritivo e qualitativo. $O$ desenvolvimento se deu pela coleta de dados através do acesso online aos bancos de dados e sistemas de busca SCIELO, PubMed e BIREME utilizando os seguintes descritores: depressão, idosos e antidepressivos. Foram selecionados artigos que se enquadraram no tema proposto e que foram publicados no período entre 2001 e 2017. Alguns livros de Farmacologia foram incluídos no estudo, como base de informações sobre os medicamentos antidepressivos. O texto foi construído abordando os principais aspectos da depressão nos pacientes idosos, os medicamentos mais prescritos no tratamento dessa doença e de que forma o profissional farmacêutico pode contribuir com a otimização deste tratamento.

\section{DEPRESSÃO EM IDOSOS}

No presente momento, destaca-se o crescimento quantitativo de pessoas com idade igual ou superior a 65 anos de idade nos países desenvolvidos. No Brasil, a representatividade desta faixa etária é de 14,5 milhões de pessoas, correspondente a $8,6 \%$ do total da população do país, com projeção para 9,7\% no ano de 2050 (Lima et al., 2016).

Cumpre esclarecer que a depressão é considerada uma das doenças mais impactantes das últimas décadas, ocorre em todas as faixas etárias, inclusive nos idosos, acarretando sentimento de tristeza e isolamento social, podendo provocar surtos e também o suicídio (Drago \& Martins, 2012). 
$\mathrm{Na}$ terceira idade, o desenvolvimento da depressão engloba os aspectos biológicos (vulnerabilidade na saúde decorrente de doenças crônicas), psicológicos (viuvez, falta de atividades sociais, e mudança de papeis) e sociais (escolaridade, pobreza, solidão e modificações no suporte social) (Soares et al., 2013).

Neste contexto, é importante diferenciar tristeza e depressão. A tristeza é um estado momentâneo causado por perdas, lembranças de momento difíceis, desilusões, fim de relacionamentos e diversas outras formas. Esses sentimentos tendem a ser passageiros, cujos sintomas vão se diminuindo com o decorrer do tempo e a pessoa consegue voltar sua rotina normal. Porém, quando esses sintomas persistem por duas semanas consecutivas e são acompanhados de apatia, desesperança, indiferença, apresentam-se sinais claros de depressão, necessitando, portanto, de ajuda médica (Lima et al., 2016).

Ademais, cabe ressaltar que a depressão é caracterizada por transtorno de humor de grande sofrimento, rodeado por perda de energia e interesse, sentimento de culpa, dificuldade de concentração, pensamentos negativos, modificação no sono e na atividade sexual, pensamentos de suicídio ou morte, alterações do apetite (ganho ou perda de peso), desamparo e diminuição ou perda de interesse em realizar atividades (Feitosa, 2014). No idoso, a depressão é apresentada de maneira atípica ou indireta, ou seja, encoberta por múltiplas e variadas queixas somáticas e associada a quadros de ansiedade (Silva, 2010).

$\mathrm{Na}$ maioria das vezes, encontram-se idosos nos quais o diagnóstico não foi corretamente efetuado, por terem uma grande dificuldade de ir ao médico ou até mesmo porque são incapazes de responderem às perguntas. Após a realização correta do diagnóstico, o idoso recupera a alegria de viver, ganha autonomia nas suas atividades cotidianas e a família reencontra uma nova pessoa que teria desistido da vida (Drago \& Martins, 2012).

Sendo assim, é importante frisar ser de extrema importância à procura de profissional competente, a fim de que seja realizado corretamente o diagnóstico, e, por consequência, tratar-se de maneira eficaz.

Epidemiologicamente estima-se que aproximadamente $15 \%$ da população na faixa etária de 60 anos ou superior, apresentam alguns sintomas da depressão, e, dentro desses, $2 \%$ do tipo grave da enfermidade, podendo atingir de $5 \%$ a $13 \%$ dos 
pacientes hospitalizados e de $12 \%$ a $16 \%$ dos residentes nas instituições de longa permanência (Coutinho, Gontiès, Araújo, \& Sá, 2003).

A incidência de depressão é mais elevada nos idosos asilares ou naqueles que se encontram em tratamento nos hospitais para internação de doentes agudos do que na comunidade. As taxas de prevalência dos sintomas depressivos nessas populações são de $31 \%$ a $23 \%$, respectivamente. Aproximadamente $13 \%$ dos idosos asilados desenvolvem episódios depressivos no meio de um ano (Coutinho et al., 2003).

As causas da depressão são variadas, podendo surgir de fatores biológicos, psicológicos e sociais. A causa biológica é a presença de depressão em outros membros da família, considerando-se predisponentes. Por sua vez, a causa psicológica é o fato de adoecer devido à morte. Por fim, as causas sociais se relacionam à perda de emprego, divórcio da família, brigas e em decorrência do abandono da família (Coutinho et al., 2003).

A depressão pode ser causada também por substâncias de abuso (como álcool), doenças infecciosas, neurológicas, metabólicas, neoplasias, câncer e endócrinas ou doenças cardiovasculares (Martinelli, 2016).

Ainda há outros fatores que podem causar a depressão nos idosos, os quais estão relacionados a perdas de amigos, cônjuge, parentes, assim como perdas financeiras, perda de status e de papeis sociais. Tais motivos levam as pessoas acreditarem que a morte não está muito longe, o que faz com que alguns idosos passem a aproveitar melhor a vida vivenciando o momento presente, enquanto outros entram em desespero e não conseguem formular mais suas perspectivas de vida (Martinelli, 2016).

O idoso por sua vez enfrenta todas as transformações causadas pela idade, além das mudanças físicas, psicológicas e sociais. A comunidade já impõe que o idoso está fora do mercado de trabalho, sem falar nas mudanças físicas que às vezes podem os deixar debilitados, algumas dessas alterações são: perda de massa óssea, os músculos tendem a diminuir de volume, a flexibilidade também diminui, além da possibilidade de sofrerem mais de hipertensão, artrose, artrite, arteriosclerose, osteoporose, entre outras doenças (Viana \&AntoniassiJunior, 2017). 
Vale acrescentar, neste contexto, que alguns medicamentos também podem causar sintomas depressivos, como os antiparkinsonianos (L-dopa, amantadina), benzodiazepínicos (diazepam e outros), anti-hipertensivos (alfa-metildopa, clonidina, nifedipina, propranolol, digoxina), além de corticóides (Stella et al., 2002).

$\mathrm{Na}$ década de 80 , foi fundada uma rede de instituições de prestação de serviços denominado "asilo", com o objetivo de oferecer aos idosos, sem vínculo com a família ou sem nenhuma condição de organizar a própria vida, a satisfação das necessidades básicas, como alimentação, moradia e convivência social (Pestana \& Santo, 2008). A instituição asilar é compreendida como a modalidade mais antiga e universal de assistência aos idosos fora do seu convívio familiar.

Os asilos são chamados atualmente de Instituições de Longa Permanência (ILPI), que é especifico para os idosos. Há uma grande procura por vagas nas instituições brasileiras, não só para os idosos com alta dependência, mas também por idosos jovens, entre 60 e 65 anos, que foram extirpados do mercado de trabalho e da proteção da família (Pestana \& Santo, 2008).

Geralmente, os asilos estão correlacionados a imagens negativas e preconceituosas, os quais são vistos como "depósito de idosos" à espera do tempo da sua morte. Na sociedade brasileira, os asilos relembram pobreza, negligência e abandono do idoso pelos seus familiares, diferenciando-se da atual concepção de Instituição de Longa Permanência para Idosos (ILPI) e de envelhecimento ativo, apontando uma nova visão de relacionamento e de atividades que buscam manter os idosos envolvidos em novas ou antigas experiências, mantendo-os dinâmicos (Hartmann Júnior \& Gomes, 2014).

A velhice é uma questão muito difícil de ser analisada, pois, as pessoas trabalham por toda sua vida, e, no final, a etapa (da vida) em que se precisa de paz e descanso, acontece o contrário. Ou seja, a saúde já não é a mesma, a comunidade critica e até mesmo a família considera o idoso uma perturbação, colocando-o em uma instituição asilar, fazendo, desse modo, nascer o sentimento de rejeição e contribuindo para aceleração do processo de envelhecimento (Oliveira, 2001).

A partir do momento que o idoso se integra ao ambiente institucional, ele passa a ter dificuldade de lidar com as perdas, por exemplo, de status e de papéis sociais, tendo que encarar problemas de saúde e de ordem econômica e diversas 
outras questões. Alguns sentem desespero, angústia, insegurança, tensão, e quanto maior for o potencial para a perda desse vínculo, mais intensa e variadas serão essas reações, podendo, por consequência, prejudicar o indivíduo (Carmo, Rangel, Ribeiro, \& Araújo, 2003). A maioria das internações de idosos acontece contra a sua vontade, o que podem desencadear fatores que intensificam a alta prevalência de depressão em instituições asilares (Gonçalves \&Lemgruber, 2016).

A instituição de longa permanência pode ser um lugar muito estressante com alta probabilidade de desencadear a depressão, pois, o idoso institucionalizado depara-se com um estilo de vida diferente do seu, precisando se adaptar com a rotina de horários, dividir seu ambiente com pessoas desconhecidas, e, além de tudo, ficar longe de seus familiares. Alguns idosos podem vir a perder sua autonomia, sua liberdade, sua identidade, fatores que intensificam a alta prevalência de depressão em instituições asilares (Souza, 2016).

De outro lado, idosos socialmente integrados e felizes nas suas relações familiares relatam um menor número de sintomas depressivos. Portanto, observa-se que a família desempenha um papel relevante para a socialização do idoso na ILPI, proporcionando efeitos benéficos sobre o bem-estar e a saúde dos mesmos, enfim, possibilitando uma melhor qualidade de vida (Souza, 2016).

\section{MEDICAMENTOS UTILIZADOS NO TRATAMENTO DA DEPRESSSÃO}

Além das causas sociais e psicológicas, a depressão também possui causas biológicas, conforme acima mencionado. De acordo com a Teoria Monoamínica da Depressão, a depressão resulta de uma diminuição patológica na neurotransmissão de serotonina e/ou norepinefrina que, dentre outras funções, desempenham papéis críticos na modulação do humor, no ciclo de sono-vigília e na motivação. Com base nessa hipótese, pode-se deduzir que o aumento na neurotransmissão da serotonina e/ou norepinefrina pode melhorar ou reverter a depressão (Golan, Tashjian Júnior, ArmstrongEhrin, \& Armstrong, 2009). Sendo assim, a ação dos antidepressivos se baseia na modulação desses neurotransmissores. 
A seguir são citados os principais medicamentos utilizados no tratamento da depressão:

\begin{tabular}{c|c}
\hline Classe do antidepressivo & Representantes \\
\hline \hline Antidepressivos tricíclicos & $\begin{array}{c}\text { Imipramina, Amitriptilina, } \\
\text { Desipramina, Nortriptilina e Clomipramina }\end{array}$ \\
\hline $\begin{array}{c}\text { Inibidores seletivos da } \\
\text { recaptação de serotonina }\end{array}$ & $\begin{array}{c}\text { Fluoxetina, Citaprolam, Paroxetina, } \\
\text { Inibidores da recaptação da } \\
\text { serotonina e da noradrenalina }\end{array}$ \\
\hline
\end{tabular}

Quadro 1- Antidepressivos e suas classes farmacológicas

Fonte: (Golan et al., 2009).

Os Antidepressivos Tricíclicos (ADT) possuem como mecanismo de ação a redução da recaptação de serotonina e norepinefrina, aumentando a disponibilidade desses neurotransmissores na fenda sináptica. Alguns possuem predominância da atividade noradrenalina e outros da serotoninérgica. Podem ainda bloquear os receptores histaminérgicos, colinérgicos e muscarínicos responsáveis pela maioria dos efeitos adversos (BRATS, 2012). Os efeitos anticolinérgicos consistem em sintomas típicos de bloqueio dos receptores muscarínicos de acetilcolina: boca seca, visão turva, confusão, náusea, vômitos, anorexia, constipação, taquicardia e retenção urinária. Já os efeitos anti-histaminérgicosincluem ganho de peso, sedação e confusão (no idoso). Por fim, os efeitos antiadrenérgicosconsistem em hipotensão ortostática, sonolência, taquicardia reflexa, e tonteira. A hipotensão ortostática constitui um risco particularmente significativo em pacientes idosos, e nesses indivíduos é necessário proceder a uma cuidadosa monitorização (Golan et al., 2009).

Apesar dos efeitos indesejados, os pacientes devem ser incentivados a continuar a terapia, pois a incidência desses efeitos vai diminuindo com o decorrer do tempo, uma vez que ocorre tolerância (BRATS, 2012).

Em geral, os antidepressivos tricíclicos possuem alta ligação às proteínas plasmáticas ( $\approx 90 \%)$, metabolismo hepático via CYP3A4, 2D6, 1A2 e 1C19, e meia vida de eliminação de 24 horas, o que facilita uma única administração diária. 
Geralmente são administrados à noite devido aos efeitos anticolinérgicos provocados, como sedação (BRATS, 2012).

A administração de barbitúricos parece diminuir os níveis dos ADT's, por causa da indução enzimática do microssômico hepático. Não é aconselhado a pacientes em uso de tricíclicos administrar barbitúricos (Silva, 2017).

Vários autores determinaram uma inibição da metabolização dos ADT com consequente aumento dos seus níveis plasmáticos pelos neurolépticos, principalmente perfenazina, clorpromazina, levomeorimazina e haloperidol. O mecanismo sugerido consiste na diminuição da hidroxilação. Os outros fatores que interferem com os níveis plasmáticos dos ADT's são mostrados no quadro abaixo (Silva, 2017).

\begin{tabular}{l|l}
\hline Diminuem & Aumentam \\
\hline Álcool & Cloranfenicol \\
\hline Barbitúricos & Corticosteroide \\
\hline Carbamazepina & Dissulfiram \\
\hline Contraceptivos orais & Fluoxetina \\
\hline Fumo & Meperidina \\
\hline Glutetimida & Metilfenidato \\
\hline Hidrato de cloral & Morfina \\
\hline
\end{tabular}

Quadro 2 - Drogas que modificam os níveisplasmáticos dos ADT

Fonte: (Silva, 2017).

Os ADT'sbloqueiam os efeitos anti-hipertensivos da guanetidina, portanto são contraindicados para os pacientes que fazem uso dessa droga (Silva, 2017).

Outra classe muito utilizada no tratamento da depressão são os Inibidores Seletivos da Recaptação de Serotonina (ISRS). Os ISRS inibem a receptação da serotonina na fenda sináptica, aumentando a liberação da monoamina e, consequentemente, a atividade serotonérgica. Por serem seletivos, não exercem ação sobre outras catecolaminas (norepinefrina e dopamina). Contudo, apresentam atividades anticolinérgicas, adrenérgica e histaminérgica, relacionadas com a 
manifestação dos efeitos adversos da classe, porém de forma menos agravante que os ADT's (BRATS, 2012).

Todos os ISRS podem ser administrados em dose única diária, pois a meia vida de eliminação varia de 20 a 30 horas. O pico plasmático ocorre 8 horas após sua administração. A ingestão com alimentos não interfere na absorção destes fármacos e o metabolismo é predominantemente hepático, via CYP 2D6 (BRATS, 2012).

Os efeitos adversos mais comuns são cefaleia, alterações em sono e nível de energia, falta de coordenação, efeitos gastrointestinais e em alguns casos podem ocorrer disfunção sexual e hiponatremia (BRATS, 2012).

Todos os ISRS inibem a CYP2D6, a fluoxetina e a paroxetina são as mais potentes. Essa propriedade dá a esses compostos potencial de interação medicamentosa, pois causa inibição da oxidação de outras drogas no sistema enzimático do fígado. A fluoxetina inibe as enzimas CYP 2D6 e 2C19, tendo potencial de transportar aumento dos níveis plasmáticos de ADT e de neurolépticos (Silva, 2017).

Deve ser evitado o uso concomitante da fluoxetina e terfenadina, pois a norfluoxetina inibe a isoenzima CYP 3A4 aumentando as concentrações plasmáticas de terfenadina, que em doses elevadas se torna uma substância cardiotóxica. A sertralina, o citalopram e o escitalopram, independentemente de serem inibidores da CYP 2D6, não parecem promover interações farmacocinéticas clinicamente significativas, e são considerados mais seguros em pacientes polimedicados (Silva, 2017).

Os representantesda classe dos Inibidores da Recaptação de Serotonina e Norepinefrina (IRSN) são a venlafaxina e a duloxetina. A venlafaxina é um potente inibidor da receptação de serotonina e da norepinefrina, havendo baixa atividade sobre a dopamina. Os efeitos adversos caracterizam-se por vômitos, náuseas, cefaleia, insônia e vertigem. Geralmente os dois primeiros sintomas tendem a desaparecer com o uso do fármaco. Em doses elevadas pode ocorrer elevação transitória dose-dependente da pressão arterial, logo, deve-se acompanhá-la durante o tratamento. Em pacientes hipertensos, o uso deve ser limitado, pois pode ocorrer sangramento gastrointestinal, constipação e distúrbios na ejaculação (BRATS, 2012). 
A duloxetina também inibe especificamente a receptação de serotonina e norepinefrina, com atividade equilibrada entre as duas monoaminas. O fármaco é metabolizado pelo fígado, portanto não está indicado para hepatopatas. Há alguns efeitos adversos, os quais são: constipação, xerostomia e náusea. Podem ocorrer ainda: diarreia, vômito, prurido, alterações do sono e disfunção sexual (BRATS, 2012).

A venlafaxina por apresentar baixa ação inibitória no citocromo P450, ter meia vida curta, grande volume de distribuição e ligação proteica baixa, possui pequeno risco de interação medicamentosa. Nesse contexto, estudos avaliando a utilização concomitante de venlafaxina com diazepam, cimetidina, lítio e vários outros agentes hipertensivos não mostraram alterações significativas dos níveis plasmáticos (Silva, 2017).

A Duloxetina pode apresentar algumas interações medicamentosas as quais são: Abciximabe, ácido salicílico, celecoxibe, clopidogrel, dalteparina, diclofenaco, dicumarol, dipiridamol, dipirona, enoxaparina, heparina, ibuprofeno, indometacina, nadroparina, tenoxicam: Risco aumentado de sangramento. Amitriptilina, clorpromazina, clomipramina, imipramina, nortriptilina: Pode ocorrer aumento nos níveis plasmáticos desses medicamentos, podendo levar a efeitos tóxicos. Citalopram, desvenlafaxina, escitalopram, fluoxetina, linezolida, carbonato de lítio: risco aumentado de desencadear síndrome serotonérgica (Santos et al., 2013).

\section{ASSISTÊNCIA FARMACÊUTICA RELACIONADA AO USO DE ANTIDEPRESSIVOS NOS IDOSOS}

O uso de antidepressivos em idosos evoluiu muito no decorrer dos anos. Desde a introdução dos ISRS no mercado, entre as décadas de 80 e 90, desenvolveuse a preferência de seu uso. Tais fármacos apresentam menor risco de reações adversas, quando comparados com os demais antidepressivos, sobretudo, os tricíclicos (Wagner, 2015). Os ISRS são geralmente bem tolerados e isentos de risco em cardiopatas, e as superdosagens são menos perigosas que as dos ADT's (Scalco, 2002).

Sendo assim, em decorrência das variações entre as doses, da duração do tratamento e da pouca ocorrência de efeitos adversos, o uso do ISRS se firmou como 
a primeira escolha para o tratamento dos pacientes idosos nessas hipóteses. Os princípios ativos mais importantes nessa linha são: cloridrato de sertralina, cloridrato de fluoxetina e o escitalopram; porém, o cloridrato de fluoxetina é o mais utilizado atualmente (Wagner, 2015).

ADT, por sua vez, atuam sobre receptores noradrenérgicos e serotonérgicos, bem como histaminérgicos, alfa-adrenérgicos, muscarínicos e dopaminérgicos, e são responsáveis por vários efeitos colaterais. Os mais ocorrentes são: hipotensão ortostática, boca seca, tremores, constipação, taquicardia, aumento dos intervalos PR e QRS no eletrocardiograma (ECG) e diminuição da pressão arterial sistólica ao levantar (Scalco, 2002).

Os tricíclicos podem causar, também, alterações cognitivas. A mais comum é o prejuízo da memória de curta duração. Em pacientes idosos e com déficits cognitivos, esse efeito pode piorar o desempenho cognitivo. Além disso, quando estão em superdosagem podem causar delírio, confusão e desorientação (Scalco, 2002).

Dentre os efeitos colaterais, o mais perigoso, devido à imprevisibilidade, parece ser a hipotensão ortostática, pois não é dose dependente e pelo eu potencial para causar consequências como quedas, síncopes, infarto do miocárdio ou acidentes vasculares cerebrais. Foram descritas quedas maiores que $20 \mathrm{mmHg}$ na pressão sistólica ao levantar com imipramina ou clomipramina, quedas de $10 \mathrm{mmHg}$ com nortriptilina, quedas maiores que $30 \mathrm{mmHg}$ em cardiopatas com imipramina e quedas de $15 \mathrm{mmHg}$ em idosos hipertensos controlados com nortriptilina (Scalco, 2002).

Em pacientes que utilizam outros medicamentos que podem aumentar a ação hipotensora, o risco de hipotensão ortostática é potencializada. Pode-se citar como exemplo neste caso os anti-hipertensivos e os neurolépticos (Fleming \&Goetten, 2005).

Metildopa associada com antidepressivos tricíclicos causa diminuição do efeito hipotensor, já se associada com lítio, ocorre toxidade pelo mesmo, pelo mecanismo de redução da excreção renal do lítio (Fleming \&Goetten, 2005).

Clonidina por via oral produz hipotensão. Quando associada com antidepressivos, inibe-se o seu efeito hipotensor. Com isso o aumento da PA (pressão arterial) ocorre na segunda semana de uso dos antidepressivos, podendo ocasionalmente se dar nos primeiros dias (Fleming \&Goetten, 2005). 
Vale destacar também os riscos associados ao uso concomitante de dois antidepressivos ou de antidepressivo com outro psicofármaco, tendo em vista que muitos desses fármacos são metabolizados pelas isoenzimas CYP2D6 e CYP3A4 (Martins, Figueiredo, Costa, \& Reis, 2015).

Os ADTs afetam a frequência cardíaca em repouso, em média de nove batimentos por minuto, por ação colinérgica. Em situações especificas, esse efeito pode ser perigoso. Aumento da frequência cardíaca em repouso significa aumento do consumo de oxigênio e do fluxo sanguíneo coronário, podendo desencadear isquemia cardíaca (Scalco, 2002).

No entanto, o uso de ADT em idosos pode ser seguro e eficaz, desde que os pacientes sejam adequadamente monitorizados. Em algumas situações os ADT's são contraindicados, como acontece no caso de pacientes com prostatismo ou arritmias. Em geral, deve-se dar preferência à nortriptilina e à desipramina por terem menos efeitos adversos e pelo fato de terem metabolismo mais rápido (Scalco, 2002).

É de bom alvitre mencionar que a Farmácia Clínica é uma área de extrema importância para o eficaz tratamento dos pacientes idosos com depressão. Ademais, não apenas destes, mas de todos os pacientes que porventura necessitarem de tratamento, uma vez que contribui para o acesso, uso racional e seguro dos medicamentos prescritos. Neste contexto, a Farmácia Clínica tende identificar problemas relacionados com medicamentos (PRM) e realizar intervenções farmacêuticas, juntamente coma equipe de saúde, facilitando uma melhora clínica dos pacientes acompanhados (Garske, Freitas, Brixner, Machado, \& Schneider, 2016).

O farmacêutico clínico, portanto, deve realizar orientações sobre os medicamentos tanto para o paciente, quanto para os profissionais da saúde, monitorar a farmacoterapia prescrita e intervir, quando for necessário, como reconciliação medicamentosa, via de administração, forma farmacêutica, posologia e administração inadequadas, bem como na substituição de medicamentosos envolvidos em interações medicamentosas, a fim de garantir a segurança do paciente (Garske et al., 2016).

Ao colaborar para a redução do número de medicamentos inadequados para osidosos e sugerir intervenções considerando as particularidades dos pacientes dessa faixa etária, o farmacêutico contribui na qualificação do cuidado ao idoso. 
A partir de toda análise acerca do tratamento da depressão em idosos, podese concluir que o farmacêutico possui atribuições de grande importância no trabalho conjunto com os profissionais da saúde, orientando-os quanto aos riscos que a farmacoterapia pode oferecer, assim como a maneira correta de medicar os pacientes, garantindo a segurança e eficácia do tratamento.

\section{CONSIDERAÇÕES FINAIS}

A depressão é uma doença cada vez mais comum na terceira idade e pode ter diversas causas. Seu tratamento se dá principalmente através da intervenção medicamentosa. Cada medicamento possui sua particularidade, logo cada paciente deve ser examinado e diagnosticado através de avaliações prévias de profissionais capacitados para posterior inserção do tipo de medicamento correto para um tratamento eficaz.

Alguns medicamentos podem trazer riscos à saúde do idoso, portanto cada caso deve ser assistido pela equipe de saúde de forma individual, de modo a minimizar qualquer tipo de falha que venha a ocorrer pelo uso indevido dos medicamentos. 


\section{REFERÊNCIAS}

Brats. (2012). Antidepressivos no transtorno depressivo maior em adultos. (18).

Carmo, H. O., Rangel, J. R. A., Ribeiro, N. A. P., \& Araújo, C. L. O. (2012). Idoso institucionalizado: o que sente, percebe e deseja? Revista Brasileira de Ciências do Envelhecimento Humano, 9(3),330-340.

Coutinho, M. P. L., Gontiès, B., Araújo, L. F., \& Sá, R. C. N. (2003). Depressão, um sofrimento sem fronteira: representações sociais entre crianças e idosos. Psico - USF, 8(2),183-192. Recuperado em 27 de junho, 2017, de http://www.scielo.br/scielo.php?pid=S1413-

$82712003000200010 \&$ script=sci_abstract\&tlng=pt.

Drago, S. M. M. S., \& Martins, R. M. L. (2012). A Depressão no Idoso. Repositório cientifico do Instituto Politecnico Portugal, 79-94. Recuperado em 15 de abril, 2017, de http://repositorio.ipv.pt/handle/10400.19/1663.

Feitosa, F. B. (2014). A depressão pela perspectiva biopsicossocial e a função protetora das habilidades sociais. Revistas Científicas de América Latina,34(2),488499. Recuperado em 23 de junho, de 2017, de http://www.scielo.br/scielo.php?pid=S141498932014000200016\&script=sci_abstract\&tlng=pt.

Fleming, I., \& Goetten, L. F. (2005). Medicamentos mais utilizados pelos idosos: implicações para a enfermagem. Arq. Ciênc. Saúde Unipar, 9(2),121-128. Recuperado em 16 de julho, 2017, de http://www.saudedireta.com.br/docsupload/1284732063184.pdf.

Garske, C. C. D., Freitas, A. P., Brixner, B., Machado, E.O., \& Schneider, A. P. H. (2016). Acompanhamento farmacoterapêutico de pacientes atendidos em pronto socorro atendimento em um hospital de ensino. Revista Saúde Santa Maria, 42(1),114-119. Recuperado em 22 julho, 2017, de https://periodicos.ufsm.br/revistasaude/article/view/21031/pdf.

Golan, D. E., Tashjian Junior, A. H., Armstrong, E. J., \& Armstrong, A. W. (2009). Princípios de farmacologia: a base fisiopatológica da farmacoterapia (2a ed.) Rio de Janeiro: Guanabara Koogan.

Gonçalves, K. C. S., \& Lemgruber, K. P. (2016). Breve histórico sobre a velhice e instituições asilares no Brasil. Psicologia E Saúde Em Debate, 2(Supl. 1),21-23.

Júnior, J., \& Gomes, G. (2014). Depressão em idosos institucionalizados: as singularidades de um sofrimento visto em sua diversidade. Revista Sociedade Brasileira Psicologia Hospitalar - RBPH, 17(1),83-105. Recuperado em 30 de junho, 2017, de http://pepsic.bvsalud.org/scielo.php?script=sci_arttext\&pid=S151608582014000200006 . 
Lima, A. M. P., Ramos, J. L. S., Bezerra, I. M. P., Rocha, R. P. B., Batista, H. M. T., Pinheiro, W. R. et al. (2016). Depressão em idosos: uma revisão sistemática da literatura. Revista Epidemiologia e Controle de Infecção,6(2),97-103. Recuperado em 22 de junho, 2017, https://online.unisc.br/seer/index.php/epidemiologia/article/viewFile/6427/5091.

Martinelli, J. (2016). Principais Sintomas da Depressão em Idosos. Recuperado em 15 de abril, 2017, de https://idosos.com.br/sintomas-depressao-idosos/.

Martins, J. M., Figueiredo, T. P., Costa, S. C., \& Reis, A. M. M. (2015). Medicamentos que podem induzir prolongamento do intervalo QT utilizados por idosos em domicílio. Revista de Ciências Farmacêuticas Básica e Aplicada, 36(2),297-305. Recuperado em 16 de julho, 2017, http://seer.fcfar.unesp.br/rcfba/index.php/rcfba/article/view/245.

Oliveira, R. S. (2001). O significado do estar asilado para o idoso. Repositório Institucional, 10-84. Recuperado em 21 de maio, 2017, de https://repositorio.ufba.br/ri/bitstream/ri/10251/1/Disserta\%C3\%A7\%C3\%A3o_Enf_R os\%C3\%A2ngela\%20Oliveira.pdf.

Pestana, L.C., \& Santo, F.H.E. (2008). As engrenagens da saúde na terceira idade: um estudo com idosos asilados. Revista da Escola de Enfermagem da USP, 42(2),268-75. Recuperado em 10 de julho, 2017, de http://www.scielo.br/scielo.php?script=sci_arttext\&pid=S0080-62342008000200009.

Santos, L., Torriani, M. S., Barros, E., Sartori, A. A. T., Camargo, A. L., Pimentel, A. L. et al. (2013). Medicamentos na prática da farmácia clínica. Porto Alegre: Artmed.

Scalco, M. Z. (2002). Tratamento de idosos com depressão utilizando tricíclicos, IMAO, ISRS e outros antidepressivos. Revista Brasileira de Psiquiatria, 24(Supl I),5563. Recuperado em 16 de julho, 2017, de http://www.scielo.br/scielo.php?pid=S151644462002000500011\&script=sci_abstract\&tlng=pt.

Silva, G. B. (2010). Caracterizando a depressão no idoso: uma revisão bibliográfica. Centro científico conhecer. Enciclopédia biosfera, 6(9),1-7. Recuperado em 26 de julho, 2017, de http://www.academia.edu/5058286.

Silva, P. (2017). Farmacologia (8ª ed.) Rio de Janeiro: Guanabara Koogan.

Soares, P. F. C., Oliveira, F. B., Freitas, E. A. F., Leite, E. S., Nogueira, J. R. F., Nóbrega, A. C. et al. (2013). Depressão em idosos assistindo nas unidades básicas de saúde. Revista enfermagem UFPE on line, 7(9),5453-5459. Recuperado em 2 de julho, 2017 , de https://periodicos.ufpe.br/revistas/revistaenfermagem/article/view/11829. 
Souza, A. P. P. (2016). Prevalência de depressão em idosos institucionalizados e sua relação ao suporte familiar. Psicologia Social. Recuperado em 10 de maio, 2017, de https://psicologado.com/atuacao/psicologia-social/prevalencia-de-depressao-emidosos-institucionalizados-e-sua-relacao-ao-suporte-familiar.

Stella, F., Gobbi, S., Corazza, D. I., \& Costa, J. L. R. (2002). Depressão no idoso: diagnóstico, tratamento e benefícios da atividade física. Centro esportivo virtual, 8(3),91-98. Recuperado em 30 de junho, 2017, de http://www.rc.unesp.br/ib/efisica/motriz/08n3/Stela.pdf.

Viana, A. \& Junior, G. (2017). Qualidade de vida em idosos praticantes de atividades físicas. Psicologia E Saúde Em Debate, 3(1), 87-98.

Wagner, G. A. (2015). Tratamento de depressão no idoso além do cloridrato de fluoxetina. Revista Saúde Pública. São Paulo. Brasil, 49,20. Recuperado em 5 de abril, 2017, de http://www.scielo.br/pdf/rsp/v49/pt_0034-8910-rsp-S003489102015049005835.pdf. 